\title{
MODELAGEM DA PERMEAÇÃO DE HIDROGÊNIO EM MEMBRANAS COMPOSTAS ${\mathrm{Pd} / \mathrm{Al}_{2} \mathrm{O}_{3}}_{3} \mathrm{COM}$ ESTRUTURA ASSIMÉTRICA DO TIPO FIBRAS OCAS
}

\author{
A.C. RIBEIRO NETO ${ }^{1}$, A. J. de ASSIS $^{1}$, M. H. M. REIS ${ }^{1}$ \\ ${ }^{1}$ Universidade Federal de Uberlândia, Departamento de Engenharia Química \\ E-mail para contato: miria@feq.ufu.br
}

\begin{abstract}
RESUMO - O hidrogênio é matéria-prima em vários processos industriais e destaca-se como combustível limpo. Este pode ser produzido em reatores com membrana, possibilitando um aumento na pureza do hidrogênio obtido e na conversão da reação. Membranas compostas de paládio suportadas em alumina com estrutura assimétrica e configuração de fibras ocas permitem maior permeabilidade de hidrogênio. Para melhor compreender o processo de permeação do hidrogênio através da membrana, este trabalho envolve a modelagem dos dados de fluxo de hidrogênio utilizando a lei de Sieverts. Dados experimentais de fluxo de hidrogênio em membranas de paládio suportadas em alumina foram utilizados para aplicação do modelo. Os resultados obtidos mostraram que, visto que a camada de Pd é menor que $3 \mu \mathrm{m}$, a difusão no seio do gás foi a etapa controladora.
\end{abstract}

\section{INTRODUÇÃO}

Hidrogênio é largamente usado na indústria de petróleo especialmente na hidrodealquilação, hidrodesulfonação e hidrocraqueamento e tem atraído recentemente a atenção como um possível carregador alternativo de energia para aliviar os problemas ambientais derivados dos combustíveis fósseis. Atualmente o hidrogênio é produzido de diversas maneiras, como eletrólise da água, reforma à vapor do metano, gasificação de carvão ou oxidação parcial do óleo ou gás natural, e a produção anual tem aumentado em passos consideráveis. Entretanto, o processo dominante hoje é a reforma a vapor do metano conforme descrito pela equação 1 :

$$
\mathrm{CH}_{4}+2 \mathrm{H}_{2} \mathrm{O} \leftrightarrow 4 \mathrm{H}_{2}+\mathrm{CO}_{2}
$$

As tecnologias empregadas para a separação de hidrogênio incluem a adsorção com variação de pressão (pressure swing adsorption), recuperação criogênica e separação com membranas. Comparada com outros métodos a tecnologia de separação com membranas possui um potencial de aplicação econômico considerável minimizando as operações unitárias e diminuindo o consumo de energia (Yun e Oyama, 2011).

Sabe-se que metais pertencentes aos Grupos III e IV (níquel, platina e paládio, por exemplo) da tabela periódica são permeáveis ao hidrogênio como citado por Hatlevik et al. (2010). Dentre estes metais, o paládio, bem como ligas de paládio, é o mais utilizado devido a sua alta permeabilidade de 


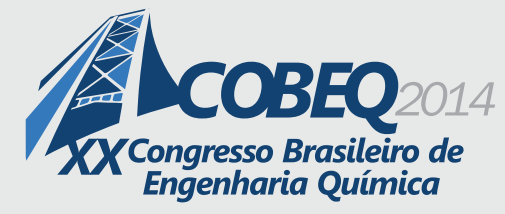

19 a 22 de outubro de 2014

Florianópolis/SC

hidrogênio, compatibilidade com misturas gasosas de hidrocarbonetos e seletividade teórica infinita para hidrogênio. O paládio pode ser ligado ainda a outros metais, como a prata, a fim de minimizar problemas de envenenamento e aumentar a vida útil da membrana.

Inicialmente, as membranas de paládio eram auto-suportadas na forma de discos ou tubos, o que representava um alto custo e ainda uma baixa permeabilidade de hidrogênio. Atualmente, pesquisas são realizadas para desenvolver suportes para membranas de paládio, com alta permeabilidade, porém garantido a resistência mecânica necessária. Devido a ampla disponibilidade, estabilidade térmica e resistência mecânica, a alumina $\left(\mathrm{Al}_{2} \mathrm{O}_{3}\right)$ é o material mais comumente utilizado como substrato para deposição de Pd como descrito por Yun e Oyama (2011). As geometrias mais utilizadas são a tubular ou plana. Porém, a aplicação de fibras ocas cerâmicas, ao invés de tubos, permite uma maior razão entre a área superficial da membrana e o volume do sistema. Ainda, a estrutura de poros assimétrica, com uma camada externa esponjosa e uma camada maior interna com filamentos, apresenta características favoráveis para uma alta permeação com uma região externa lisa para deposição do metal, como descrito por Hatim et al. (2011).

O mecanismo de permeação de hidrogênio através de membranas de paládio tem sido estudado extensivamente e quando previsto que a difusão atômica do hidrogênio através do seio do metal é a etapa determinante, o fluxo de hidrogênio através da membrana pode ser descrito pela combinação da lei de Fick com a Lei de Sieverts (Yun and Oyama, 2011).

De acordo com a Lei de Sieverts, o fluxo de hidrogênio é proporcional à diferença de pressão elevada a um expoente $n$. A literatura relata valores para o expoente $n$ da lei de Sieverts entre 0,5 e 1 . Quando o fluxo é limitado pela difusão de hidrogênio no seio da membrana, valores de 0,5 para $n$ são frequentemente encontrados (Yun and Oyama, 2011). Valores de $n$ maiores que 0,5 são atribuídos ao aumento da influência dos efeitos de superfície (adsorção e dessorção) e de transferência de massa, usualmente em membranas mais finas (Uemiya et al., 1999; Hatim et al., 2010; Boeltken et al., 2012). Uemiya et al. (1991) realizaram experimentos de permeação de hidrogênio e verificaram que o melhor ajuste do fluxo com a pressão foi obtido com o valor de $n$ igual a 0,76. Yun e Oyama (2011) analisaram diferentes valores de $n$ como função da espessura de Pd e concluíram que quando a espessura de Pd é menor que $5 \mu \mathrm{m}$ o valor de $n$ deve ser igual a 1, uma vez que a permeação de $\mathrm{H}_{2}$ é mais rápida e a transferência de massa torna-se a etapa controladora ao invés da difusão no seio do metal. Para membranas compostas e ultrafinas é preciso verificar o ajuste dos dados experimentais com a lei de Sieverts.

Com base no exposto, o objetivo deste trabalho é ajustar os parâmetros da equação de Sieverts com dados experimentais de fluxo de hidrogênio em membranas compostas de paládio suportado em alumina com estrutura assimétrica.

\section{METODOLOGIA}

\subsection{Dados experimentais de fluxo de hidrogênio}

Os dados experimentais utilizados neste trabalho foram obtidos pela permeação de hidrogênio 


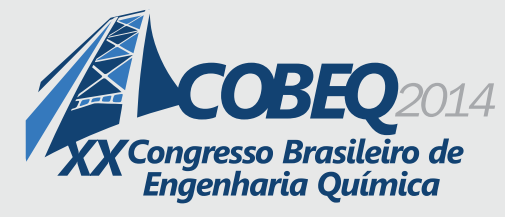

em membranas compostas formadas pela deposição de paládio sobre fibras ocas de alumina com estrutura assimétrica.

As fibras ocas assimétricas de alumina foram fabricadas utilizando-se o método de inversão e sinterização, de acordo com Kingsbury e Li (2009). Como material cerâmico utilizou-se óxido de alumínio em pó $\left(1.0 \mu \mathrm{m}\right.$, alpha, $99.9 \%$ base metálica, área superficial $\left.6-8 \mathrm{~m}^{2} \mathrm{~g}^{-1}\right)$ fornecido pela Alfa Aesar (a Johnson Matthey company). Primeiramente, o aditivo (Arlacel P135, Uniqema, UK) foi dissolvido no solvente (N-methyl-2-pyrro-lidone, HPLC grade, Rathbone) e então adicionou-se 63\% (em base mássica) de óxido de alumínio. Esta mistura foi agitada por no mínimo $48 \mathrm{~h}$ em um agitador rotativo. Em seguida, adicionou-se o polímero aglutinante (Polyethersulfone, PESf, Radal A-300, Ameco Performance, USA) em concentração de 6,3\% (base mássica) e a suspensão resultante foi agitada por mais $48 \mathrm{~h}$. Antes do processo de fiação, a suspensão cerâmica foi desgaseificada sob vácuo por cerca de $4 \mathrm{~h}$. O processo de fiação das fibras foi feito em uma peça extrusora (diâmetro externo $3 \mathrm{~mm}$, diâmetro interno $1,2 \mathrm{~mm}$ ) com dois canais através dos quais escoam a solução polimérica e o líquido interno (água) responsável pela precipitação da solução e pela manutenção do lúmen interno da fibra nascente. Ao sair da extrusora, a fibra nascente foi colocada diretamente em contato com o banho externo de precipitação. Água pura foi utilizada com coagulante externo. Antes do processo de sinterização em mufla, as fibras foram distendidas manualmente e secas em temperatura ambiente durante $48 \mathrm{~h}$. Em seguida, as fibras foram sinterizadas em mufla tubular (Elite, Model TSH $17 / 75 / 450$ ) a 1300 ou $1400^{\circ} \mathrm{C}$.

Sobre estes suportes foi depositada um ou duas camadas de paládio, pelo método de eletrodeposição, conforme descrito em Paglieri e Way (2002) e Mardilovich et al. (1998). Antes da deposição metálica, a superfície da fibra era ativada com núcleos de paládio pelo processo de sensibilização com solução de cloreto de estanho $\left(\mathrm{SnCl}_{2}\right)$ seguido da ativação com solução de cloreto de paládio $\left(\mathrm{PdCl}_{2}\right)$. Após o procedimento de sensitização/ativação, os substratos eram submersos na solução de $\mathrm{Pd}\left(\mathrm{NH}_{3}\right) 4 \mathrm{Cl}_{2}-\mathrm{H}_{2} \mathrm{O}$ em banho a $60^{\circ} \mathrm{C}$. A quantidade de solução utilizada por área superficial de fibra foi fixada em 3,5 $\mathrm{mL}$ por $\mathrm{cm}^{2}$ de fibra, conforme sugerido por Mardilovich et al. (1998). Utilizou-se solução aquosa de hidrazina $\left(\mathrm{N}_{2} \mathrm{H}_{4}\right)$ 1M como agente redutor. A quantidade de hidrazina adicionada foi calculada de maneira que sua concentração no banho de deposição fosse de $5,6 \mathrm{~mL} \mathrm{~L}^{-1}$. A solução de deposição foi homogeneizada borbulhando ar durante o procedimento. As fibras foram mantidas na solução de deposição durante $1 \mathrm{~h}$. O procedimento foi executado uma ou duas vezes, dependendo da espessura de Pd requerida.

As condições de preparo e a nomenclatura utilizada para cada membrana são apresentadas na Tabela 1. Para cada membrana, os experimentos de permeabilidade de hidrogênio foram realizados nas temperaturas de $450,400,350$ e $300^{\circ} \mathrm{C}$ e em pressões de 90 a $180 \mathrm{kPa}$. O fluxo de permeado foi registrado utilizando um medidor de bolha, sem a utilização de gás de arraste. 
Tabela 1 - Condições experimentais de preparo das membranas compostas de paládio.

\begin{tabular}{lll}
\hline Membrana & Temperatura de sinterização do suporte $\left({ }^{\circ} \mathrm{C}\right)$ & Número de camadas de paládio \\
\hline HF1 1300 / 1Pd & 1300 & 1 \\
HF1 1300 / 2Pd & 1300 & 2 \\
HF1 1400 / 1Pd & 1400 & 1 \\
HF1 1400 / 2Pd & 1400 & 2 \\
\hline
\end{tabular}

\subsection{Modelagem do fluxo de hidrogênio}

O fluxo de hidrogênio pela difusão através da membrana pode ser descrito pela primeira lei de Fick da difusão (Yun e Oyama, 2011), como mostra a Equação 2.

$$
J_{H_{2}}=-D_{H_{2}} \nabla C_{H_{2}}
$$

Onde, $J_{H_{2}}$ é o fluxo de hidrogênio, $D_{H_{2}}$ é o coeficiente de difusão e o $\nabla C_{H_{2}}$ é o gradiente da concentração de hidrogênio.

Quando a difusão no interior do metal é a etapa determinante, a lei de Sieverts, conforme descrito pela Equação 3, pode ser aplicada.

$$
S_{H}=\frac{C_{H_{2}}}{P_{H_{2}}{ }^{n}}
$$

Onde $\mathrm{S}_{\mathrm{H}}$ é a constante de solubilidade e $P_{\mathrm{H}_{2}}$ é a pressão parcial de hidrogênio.

Quando a concentração é substituída na primeira lei de Fick chega-se a Equação 4.

$$
J_{H_{2}}=-D_{H_{2}} S_{H} \frac{\Delta P^{n}}{L}=K \frac{P_{1}^{n}-P_{2}^{n}}{L}
$$

Onde $\mathrm{P}_{1}$ é a pressão no lado de maior pressão da membrana e $\mathrm{P}_{2}$ é a pressão do lado de menor pressão, L é a espessura da membrana e $K$ é coeficiente ou constante de permeabilidade.

Nesta expressão o valor do expoente da pressão $n$ varia de 0,5 a 1 dependendo de qual é a etapa determinante para a permeação de $\mathrm{H}_{2}$ pela membrana (Yun e Oyama, 2011).

A equação (4) foi ajustada aos dados experimentais e os valores de $K$ e $n$ foram obtidos. $\mathrm{O}$ ajuste foi realizado utilizando o método de Levenberg-Marquardt.

\section{RESULTADOS E DISCUSSÕES}

Os dados experimentais de fluxo de hidrogênio em função da pressão foram obtidos nas temperaturas de $450,400,350$ e $300^{\circ} \mathrm{C}$. A deposição de uma ou duas camadas de hidrogênio resultaram em membranas com espessuras de aproximadamente 1 e $3 \mu \mathrm{m}$, respectivamente. Esta 


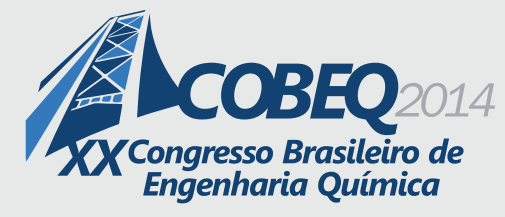

19 a 22 de outubro de 2014

Florianópolis/SC

espessura foi verificada a partir de imagens de microscopia eletrônica de varredura (MEV). A Tabela 2 apresenta os resultados obtidos para $K$ e $\mathrm{n}$ de acordo com o ajuste dos dados experimentais de fluxo em função da pressão à Equação 4.

Tabela 2 - Valores obtidos para $K$ e $n$ a partir do ajuste dos dados experimentais à equação de Sieverts.

\begin{tabular}{|c|c|c|c|}
\hline $\begin{array}{l}\text { Temperatura do } \\
\text { experimento }\left({ }^{\circ} \mathrm{C}\right)\end{array}$ & Membrana utilizada & $K\left(\mathrm{~mol} \mathrm{~m}^{-1} \mathrm{~s}^{-1} \mathbf{P a}^{\mathrm{n}}\right)$ & $n$ \\
\hline \multirow[t]{4}{*}{450} & HF1 $1300 / 1 P d$ & $3,2 \times 10^{-6}$ & 2,10 \\
\hline & $\mathrm{HF} 11300 / 2 \mathrm{Pd}$ & $1,0 \times 10^{-6}$ & 2,21 \\
\hline & $\mathrm{HF} 11400 / 1 \mathrm{Pd}$ & $1,3 \times 10^{-6}$ & 2,12 \\
\hline & $\mathrm{HF} 11400 / 2 \mathrm{Pd}$ & $5,7 \times 10^{-10}$ & 2,59 \\
\hline \multirow[t]{4}{*}{400} & $\mathrm{HF} 11300 / 1 \mathrm{Pd}$ & $3,2 \times 10^{-6}$ & 2,10 \\
\hline & $\mathrm{HF} 11300 / 2 \mathrm{Pd}$ & $2,0 \times 10^{-6}$ & 2,14 \\
\hline & HF1 $1400 / 1 \mathrm{Pd}$ & $7,9 \times 10^{-7}$ & 2,15 \\
\hline & $\mathrm{HF} 11400 / 2 \mathrm{Pd}$ & $5,7 \times 10^{-8}$ & 2,23 \\
\hline \multirow[t]{4}{*}{350} & HF1 $1300 / 1 P d$ & $2,8 \times 10^{-6}$ & 2,10 \\
\hline & $\mathrm{HF} 11300 / 2 \mathrm{Pd}$ & $1,1 \times 10^{-7}$ & 2,17 \\
\hline & HF1 $1400 / 1 \mathrm{Pd}$ & $7,5 \times 10^{-7}$ & 2,14 \\
\hline & $\mathrm{HF} 11400 / 2 \mathrm{Pd}$ & $3,3 \times 10^{-8}$ & 2,25 \\
\hline \multirow[t]{3}{*}{300} & HF1 $1300 / 1 \mathrm{Pd}$ & $2,5 \times 10^{-6}$ & 2,10 \\
\hline & $\mathrm{HF} 11300 / 2 \mathrm{Pd}$ & $9,3 \times 10^{-8}$ & 2,20 \\
\hline & $\mathrm{HF} 11400 / 2 \mathrm{Pd}$ & $2,5 \times 10^{-9}$ & 2,42 \\
\hline
\end{tabular}

Os valores de $K$ ajustados com os dados experimentais deste trabalho estão de acordo com valores reportados na literatura (Collins e Way, 1993). De acordo com Collins e Way (1993) os valores do expoente $n$ diminuem com o aumento de temperatura de realização do experimento para membranas de maiores espessuras (aproximadamente $20 \mu \mathrm{m}$ ). Entretanto, este comportamento não foi verificado a rigor nos experimentos deste trabalho. Os valores de $n$ estão próximos de $2,3 \pm 0,3$, variando de forma independente com a diminuição da temperatura do experimento. Além, de acordo com a literatura, os valores de $n$ deveriam estar entre 1 e 0,5 (Yun e Oyama, 2011). Collins e Way (1993) obtiveram valores de $n$ entre 0,526 e 0,622 para membranas de paládio suportadas em tubos cerâmicos de alumina com diferentes espessuras e em experimentos realizados a diferentes temperaturas. Hatim et al. (2011) ajustaram os dados de fluxo de hidrogênio em membranas de paládio suportados em fibras ocas de alumina com estrutura assimétrica com a variação de pressão elevada a um expoente de 0,5. Contudo, este expoente não foi otimizado no trabalho de Hatim et al. (2011).

Os maiores valores de $n$ encontrados neste trabalho podem estar associados a problemas operacionais, bem como na morfologia da membrana utilizada. Para avaliar esta questão, a Figura 1 apresenta os dados experimentais de fluxo de hidrogênio como função da pressão para as diversas membranas compostas avaliadas neste trabalho. 


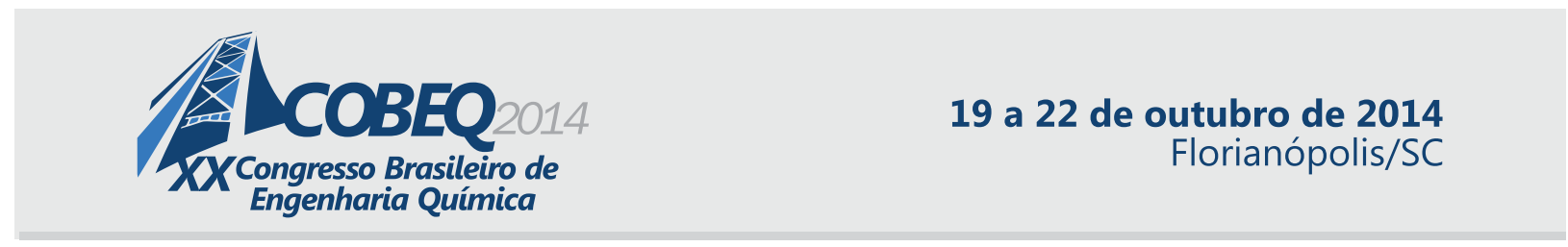

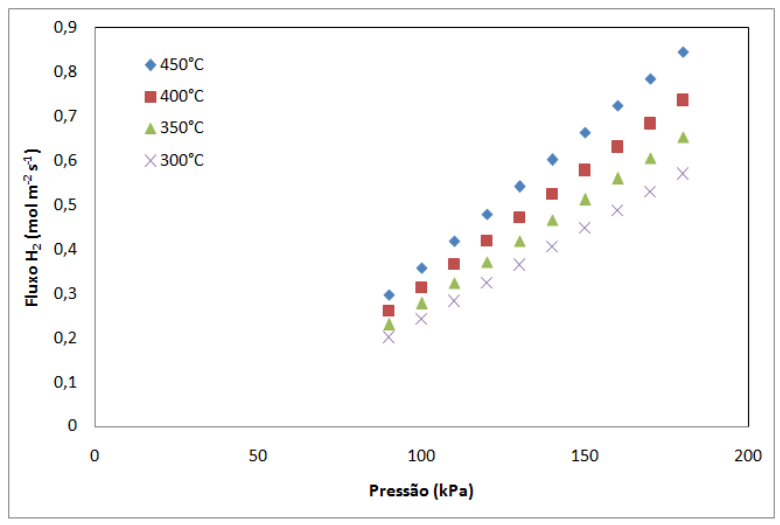

(a)

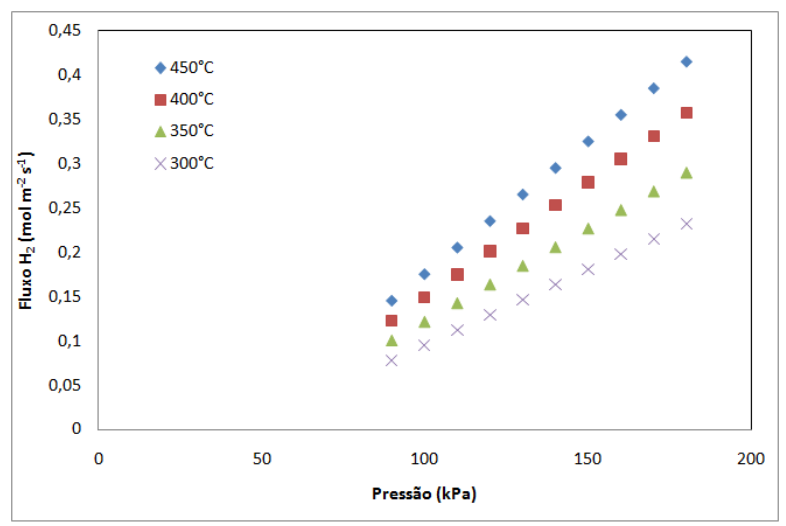

(c)

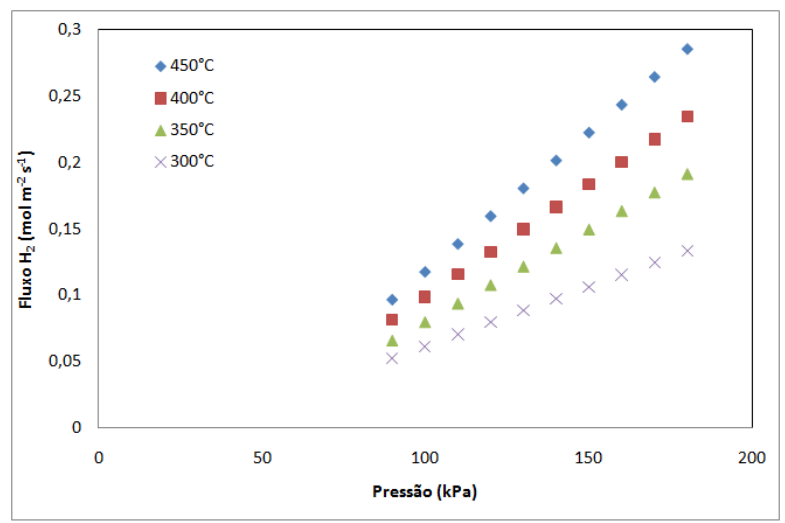

(b)

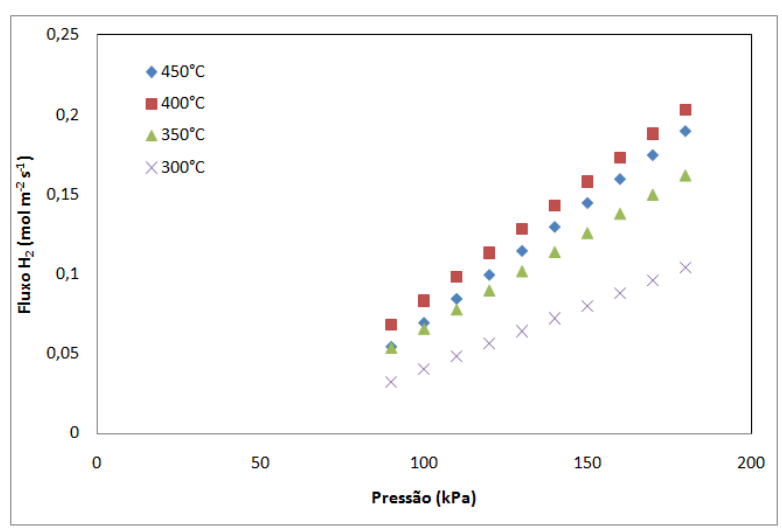

(d)

Figura 1 - Dados experimentais de fluxo de hidrogênio como função da pressão para as membranas (a) HF1 1300 / 1Pd; (b) HF1 1300 / 2Pd; (c) HF1 1400 / 1Pd; (d) HF1 1400 / 2Pd.

De acordo com a Equação 4, o fluxo deve tender a zero quando a pressão tende a zero, ou seja, o intercepto do gráfico fluxo de hidrogênio em função da pressão deve ser no ponto $(0,0)$. Contudo, verifica-se na Figura 1 que para as membranas HF1 1300/1Pd, HF1 1300/2P, HF11 1400/1Pd e HF1

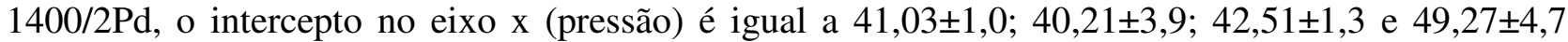
$\mathrm{kPa}$, respectivamente. $\mathrm{O}$ desvio calculado considera os dados em todas as temperaturas para cada membrana. Nota-se que este intercepto é aproximadamente o mesmo para as diversas membranas apresentadas (média de 43,26 kPa com um desvio padrão de 4,12 kPa) e não é função da temperatura do experimento. $\mathrm{O}$ fato dos dados de fluxo dos experimentos realizados não interceptarem o ponto $(0,0)$ do gráfico fluxo versus pressão deve estar levando os valores de $n$ na Equação 4 para valores maiores que 1. Pode-se inferir a partir deste comportamento que existe uma resistência adicional que faz com que o fluxo não tenda a zero quando a pressão é zero. Pizzi et al. (2008) observaram que os dados de fluxo não interceptam a origem do gráfico de pressão versus fluxo devido ao não tratamento prévio com ar na membrana, o que ocasiona um valor crítico de força motriz que tem que ser superado para atingir-se o fluxo de permeado verdadeiro. Pizzi et al. (2008) sugerem passar um fluxo de ar na superfície da membrana por $2 \mathrm{~h}$ a $400^{\circ} \mathrm{C}$ para remover contaminantes carbonáceos presentes 


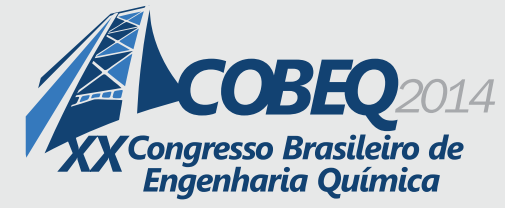

19 a 22 de outubro de 2014

Florianópolis/SC

na superfície da membrana. Vale ressaltar que nos testes realizados neste trabalho fez-se o aquecimento do sistema contendo a membrana até $450^{\circ} \mathrm{C}$ com purga de argônio a aproximadamente $1 \mathrm{ml} \mathrm{s}^{-1}$. A $450^{\circ} \mathrm{C}$, o sistema foi pressurizado a 1 bar em hidrogênio e permaneceu nesta condição por $2 \mathrm{~h}$. Trabalhos futuros serão realizados para verificar se a resistência adicional verificada nestes experimentos é eliminada pelo tratamento da membrana com ar. Vale ainda ressaltar que as espessuras das membranas utilizadas neste trabalho são menores que as reportadas na literatura e este fato pode também estar influenciando no ajuste obtido.

\section{CONCLUSÃO}

Neste trabalho, verificou-se o ajuste de dados experimentais de fluxo de hidrogênio em membranas compostas de paládio com a lei de Sieverts. O ajuste obtido mostrou que os valores da constante de permeabilidade variam de $5,7 \times 10^{-10}\left(\mathrm{~mol} \mathrm{~m}^{-1} \mathrm{~s}^{-1} \mathrm{~Pa}^{\mathrm{n}}\right)$ a $3,2 \times 10^{-6}\left(\mathrm{~mol} \mathrm{~m}^{-1} \mathrm{~s}^{-1} \mathrm{~Pa}^{\mathrm{n}}\right)$, sendo função da membrana utilizada. Os valores do expoente da lei de Sieverts para pressão $n$ ficaram em torno de 2. Este valor é a maior do que o recomendado pela literatura (entre 0,5 e 1). A análise do comportamento dos dados experimentais revelou que existe uma resistência adicional que dificulta o ajuste dos dados experimentais com a pressão com coeficiente linear igual a zero (equação 4). É preciso ainda inferir sobre a causa da ocorrência desta resistência adicional (resistência do suporte, sujidades na membrana, espessura da membrana, entre outros).

\section{AGRADECIMENTOS}

Os autores agradecem ao CNPq, CAPES e FAPEMIG pelo apoio financeiro.

\section{REFERÊNCIAS}

BOELTKEN, T., BELIMOV, M., PFEIFER, P., PETERS, T.A., BREDESEN, R., DITTMEYER, R., Fabrication and testing of a planar microstructured concept module with integrated palladium membranes. Chemical Engineering and Processing v. 67, p. 136-147, 2013.

COLLINS J. P., WAY J. D., Preparation and characterization of a composite palladium-ceramic membrane. Industrial Engineering Chemistry. v. 32, p. 3006-3013,1993.

HATIM, M.D.I., TAN, X.Y., WU, Z.T., LI, K., (2011). Pd/Al2O3 composite hollow fibre membranes: Effect of substrate resistances on H-2 permeation properties. Chemical Engineering Science, v. 66, p. 1150-1158, 2011.

HATLEVIK, O., GADE, S.K., KEELING, M.K., THOEN, P.M., DAVIDSON, A.P., WAY, J.D., Palladium and palladium alloy membranes for hydrogen separation and production: History, fabrication strategies, and current performance. Separation and Purification Technology, v. 73, p. 59-64, 2010. 
KINGSBURY, B.F.K., LI, K., A morphological study of ceramic hollow fibre membranes. Journal of Membrane Science, v. 328 ,p. 134-140, 2009.

MARDILOVICH, P.P., SHE, Y., MA, Y.H., REI, M.H., Defect-free palladium membranes on porous stainless-steel support. Aiche Journal, v. 44, p. 310-322, 1998.

PAGLIERI, S.N., WAY, J.D., Innovations in palladium membrane research. Separation and Purification Methods, v. 31, p. 1-169, 2002.

PIZZI, D., WORTH, R., BASCHETTI, M.G., SARTI, G.C., NODA, K., Hydrogen permeability of $2.5 \mathrm{mu}$ m palladium-silver membranes deposited on ceramic supports. Journal of Membrane Science, v. 325, p. 446-453, 2008.

SHU, J., GRANDJEAN, B.P.A., KALIAGUINE, S., (1995). Asymmetric Pd-Ag Stainless-Steel Catalytic Membranes for Methane Steam Reforming. Catalysis Today, v. 25, p. 327-332, 1995.

UEMIYA, S., SATO, N., ANDO, H., MATSUDA, T., KIKUCHI, E., (1991). The water gas shift reaction assisted by a palladium membrane reactor . Industrial Engineering Chemistry, v. 67, p. 223-230, 1991. 